\title{
Linear equations on real algebraic surfaces
}

\author{
Wojciech Kucharz and Krzysztof Kurdyka
}

\begin{abstract}
We prove that if a linear equation, whose coefficients are continuous rational functions on a nonsingular real algebraic surface, has a continuous solution, then it also has a continuous rational solution. This is known to fail in higher dimensions.
\end{abstract}

Key words. Linear equation, continuous rational solution, real algebraic variety.

Mathematics subject classification (2010). 14P25, 14E05, 26C15, $13 \mathrm{~A} 15$.

\section{Introduction}

Fefferman and Kollár [5] study the following problem. Given continuous functions $f_{1}, \ldots, f_{r}$ on $\mathbb{R}^{n}$, which continuous functions $\varphi$ can be written in the form

$$
\varphi=\varphi_{1} f_{1}+\cdots+\varphi_{r} f_{r}
$$

where the $\varphi_{i}$ are continuous functions on $\mathbb{R}^{n}$ ? Moreover, if $\varphi$ and the $f_{i}$ have some regularity properties, can one choose the $\varphi_{i}$ to have the same (or weaker) regularity properties? In other words, the questions are about solutions of linear equations of the form

$$
f_{1} y_{1}+\cdots+f_{r} y_{r}=\varphi
$$

The problem is hard even if $\varphi$ and the $f_{i}$ are polynomial functions. In [5], two different ways to solve the problem are presented: the Glaeser-Michael method and the algebraic geometry approach. Each of them consists of a rather complex procedure and it does not seem possible to give a concise answer in general.

In this note we settle the problem in a simple manner, assuming that $n=2$ and the $f_{i}$ are continuous rational functions. Actually, our results are more general and settle the corresponding problem for functions defined on any nonsingular real algebraic surface.

A complex version of the problem under consideration was studied by Brenner [3], Epstein and Hochster [4], and Kollár [9].

Convention 1.1. By a function we always mean a real-valued function.

Notation 1.2. If $f_{1}, \ldots, f_{r}$ are functions defined on some set $S$, then

$$
Z\left(f_{1}, \ldots, f_{r}\right):=\left\{x \in S \mid f_{1}(x)=0, \ldots, f_{r}(x)=0\right\} .
$$

The first author was partially supported by the National Science Centre (Poland), under grant number 2014/15/B/ST1/00046. He also acknowledges with gratitude support and hospitality of the Max-PlanckInstitut für Mathematik in Bonn.

The second author was partially supported by ANR project STAAVF (France). 
We now recall the pointwise test (or PT for short) introduced in [5, p. 235].

Definition 1.3. Let $\Omega$ be a metric space and let $f_{1}, \ldots, f_{r}$ be continuous functions on $\Omega$. We say that a continuous function $\varphi$ on $\Omega$ satisfies the PT for the functions $f_{i}$ if for every point $p \in \Omega$, the following two equivalent conditions hold:

(a) The function $\varphi$ can be written as

$$
\varphi=\psi_{1}^{(p)} f_{1}+\cdots+\psi_{r}^{(p)} f_{r}
$$

where the $\psi_{i}^{(p)}$ are functions on $\Omega$ that are continuous at $p$.

(b) The function $\varphi$ can be written as

$$
\varphi=\varphi^{(p)}+c_{1}^{(p)} f_{1}+\cdots+c_{r}^{(p)} f_{r}
$$

where $c_{i}^{(p)} \in \mathbb{R}$ and the functions $A_{i}^{(p)}$ defined by

$$
A_{i}^{(p)}=\frac{\varphi^{(p)} f_{i}}{f_{1}^{2}+\cdots+f_{r}^{2}} \quad \text { on } \Omega \backslash Z^{(p)} \quad \text { and } \quad A_{i}^{(p)}=0 \quad \text { on } Z^{(p)},
$$

with $Z^{(p)}:=Z\left(f_{1}, \ldots, f_{r}\right) \cup\{p\}$, are continuous at $p$.

Note that conditions (a) and (b) are indeed equivalent. If (a) holds, then so does (b) with

$$
\varphi^{(p)}=\left(\psi_{1}^{(p)}-\psi_{1}^{(p)}(p)\right) f_{1}+\cdots+\left(\psi_{r}^{(p)}-\psi_{r}^{(p)}(p)\right) f_{r} \quad \text { and } \quad c_{i}^{(p)}=\psi_{i}^{(p)}(p) .
$$

Conversely, (b) implies (a) with $\psi_{i}^{(p)}=c_{i}^{(p)}+A_{i}^{(p)}$.

Clearly, the PT is a basic necessary condition for existence of continuous functions $\varphi_{1}, \ldots, \varphi_{r}$ on $\Omega$ satisfying $\varphi=\varphi_{1} f_{1}+\cdots+\varphi_{r} f_{r}$.

For background on real algebraic geometry the reader may consult [2]. By a real algebraic variety we mean a locally ringed space isomorphic to an algebraic subset of $\mathbb{R}^{n}$, for some $n$, endowed with the Zariski topology and the sheaf of regular functions (such an object is called an affine real algebraic variety in [2]). Recall that any quasi-projective real algebraic variety is a real algebraic variety in the sense just defined, cf. [2, Prop. 3.2.10, Thm. 3.4.4]. Each real algebraic variety carries also the Euclidean topology, which is determined by the usual metric on $\mathbb{R}$. Unless explicitly stated otherwise, all topological notions relating to real algebraic varieties will refer to the Euclidean topology.

We say that a function $f$ defined on a real algebraic variety $X$ is continuous rational if it is continuous on $X$ and regular on some Zariski open dense subset of $X$. We denote by $P(f)$ the smallest Zariski closed subset of $X$ such that $f$ is regular on $X \backslash P(f)$. The continuous rational functions form a subring of the ring of all continuous functions on $X$. Any regular function on $X$ is continuous rational. The converse does not hold in general, even if $X$ is nonsingular.

Example 1.4. The function $f$ on $\mathbb{R}^{2}$, defined by

$$
f(x, y)=\frac{x^{3}}{x^{2}+y^{2}} \quad \text { for }(x, y) \neq(0,0) \quad \text { and } \quad f(0,0)=0,
$$

is continuous rational but it is not regular; in fact, $P(f)=\{(0,0)\}$. 
Recently, continuous rational functions have attracted a lot of attention, cf. [1, 6, 7, 10, 11, 12, 13, 14, 15, 16, 17, 18, 19, 20]. On nonsingular varieties they coincide with regulous functions introduced by Fichou, Huisman, Mangolte and Monnier [6].

Our first result, to be proved in Section 2, is the following.

Theorem 1.5. Let $X$ be a nonsingular real algebraic surface and let $f_{1}, \ldots, f_{r}$ be continuous rational functions on $X$. For a continuous function $\varphi$ on $X$, the following conditions are equivalent:

(a) The function $\varphi$ can be written in the form

$$
\varphi=\varphi_{1} f_{1}+\cdots+\varphi_{r} f_{r}
$$

where the $\varphi_{i}$ are continuous functions on $X$.

(b) The function $\varphi$ satisfies the PT for the $f_{i}$.

An example of Hochster [5, p. 236], which involves simple polynomial functions on $\mathbb{R}^{3}$, shows that Theorem 1.5 cannot be extended to varieties of higher dimension.

In Section 3 we prove the following.

Theorem 1.6. Let $X$ be a nonsingular real algebraic surface and let $f_{1}, \ldots, f_{r}$ be continuous rational functions on $X$. For a continuous rational function $\varphi$ on $X$, the following conditions are equivalent:

(a) The function $\varphi$ can be written in the form

$$
\varphi=\varphi_{1} f_{1}+\cdots+\varphi_{r} f_{r}
$$

where the $\varphi_{i}$ are continuous rational functions on $X$.

(b) The function $\varphi$ satisfies the PT for the $f_{i}$.

Furthermore, if (b) holds, then the $\varphi_{i}$ in (a) can be chosen so that $P\left(\varphi_{i}\right)$ is a finite set contained in $Z\left(f_{1}, \ldots, f_{r}\right) \cup P\left(f_{1}\right) \cup \ldots \cup P\left(f_{r}\right) \cup P(\varphi)$.

As a straightforward consequence we get

Corollary 1.7. Let $X$ be a nonsingular real algebraic surface and let $f_{1}, \ldots, f_{r}$ be continuous rational functions on $X$. For a continuous rational function $\varphi$ on $X$, the following conditions are equivalent:

(a) The function $\varphi$ can be written in the form

$$
\varphi=\varphi_{1} f_{1}+\cdots+\varphi_{r} f_{r}
$$

where the $\varphi_{i}$ are continuous rational functions on $X$.

(b) The function $\varphi$ can be written in the form

$$
\varphi=\psi_{1} f_{1}+\cdots+\psi_{r} f_{r},
$$

where the $\psi_{i}$ are continuous functions on $X$. 
Corollary 1.7 cannot be extended to varieties of higher dimension. A relevant example, involving polynomial functions on $\mathbb{R}^{3}$, is provided by Kollár and Nowak [10, Example 6]. Furthermore, the argument used in [10, Example 6] shows that Corollary 1.7 does not hold for the singular real algebraic surface $S \subset \mathbb{R}^{3}$ that appears there.

We conclude this section with an example.

Example 1.8. Consider the functions $f_{1}(x, y)=x^{3}, f_{2}(x, y)=y^{3}, \varphi(x, y)=x^{2} y^{2}$ on $\mathbb{R}^{2}$. We have

$$
\varphi=\varphi_{1} f_{1}+\varphi_{2} f_{2}
$$

where $\varphi_{1}, \varphi_{2}$ are continuous rational functions on $\mathbb{R}^{2}$ defined by

$$
\begin{array}{ll}
\varphi_{1}(x, y)=\frac{x^{5} y^{2}}{x^{6}+y^{6}} \quad \text { for }(x, y) \neq(0,0), \quad \varphi_{1}(0,0)=0, \\
\varphi_{2}(x, y)=\frac{x^{2} y^{5}}{x^{6}+y^{6}} \quad \text { for }(x, y) \neq(0,0), \quad \varphi_{2}(0,0)=0 .
\end{array}
$$

However, $\varphi$ cannot be written as a linear combination of $f_{1}$ and $f_{2}$ with coefficients that are regular (or $\mathcal{C}^{\infty}$ ) functions on $\mathbb{R}^{2}$, as can be seen by comparing the Taylor's expansions at $(0,0)$.

\section{Continuous solutions}

We begin with some preliminary results.

Lemma 2.1. Let $\Omega$ be a metric space and let $f_{1}, \ldots, f_{r}, \varphi$ be continuous functions on $\Omega$ such that the set $Z\left(f_{1}, \ldots, f_{r}\right)$ is nowhere dense in $\Omega$ and $\varphi$ satisfies the PT for the $f_{i}$. Assume that $f_{i}=g g_{i}$, where $g$ and the $g_{i}$ are continuous functions on $\Omega$. Then there exists a unique continuous function $\psi$ on $\Omega$ such that $\varphi=g \psi$. Furthermore, $\psi$ satisfies the PT for the $g_{i}$.

Proof. Note that the set $Z(g)$ is nowhere dense in $\Omega$. To prove existence of $\psi$ (uniqueness is then automatic) it suffices to show that for every point $p \in \Omega$ the limit

$$
\lim _{x \rightarrow p} \frac{\varphi(x)}{g(x)}, \quad \text { where } x \in \Omega \backslash Z(g)
$$

exists. This readily follows since $\varphi$ can be written as

$$
\varphi=\psi_{1}^{(p)} f_{1}+\cdots+\psi_{r}^{(p)} f_{r}=g\left(\psi^{(p)} g_{1}+\cdots+\psi_{r}^{(p)} g_{r}\right),
$$

where the $\psi_{i}^{(p)}$ are functions on $\Omega$ that are continuous at $p$.

It remains to prove that $\psi$ satisfies the PT for the $g_{i}$. We set $Z^{(p)}:=Z\left(f_{1}, \ldots, f_{r}\right) \cup\{p\}$ and write $\varphi$ in the form

$$
\varphi=\varphi^{(p)}+c_{1}^{(p)} f_{1}+\cdots+c_{r}^{(p)} f_{r}=\varphi^{(p)}+g\left(c_{1}^{(p)} g_{1}+\cdots+c_{r}^{(p)} g_{r}\right),
$$

where $c_{i}^{(p)} \in \mathbb{R}$ and the functions $A_{i}^{(p)}$, defined by

$$
A_{i}^{(p)}=\frac{\varphi^{(p)} f_{i}}{f_{1}^{2}+\cdots+f_{r}^{2}} \quad \text { on } \Omega \backslash Z^{(p)} \quad \text { and } \quad A_{i}^{(p)}=0 \quad \text { on } Z^{(p)} \text {, }
$$

are continuous at $p$. Defining $\psi^{(p)}$ by

$$
\psi=\psi^{(p)}+c_{1}^{(p)} g_{1}+\cdots+c_{r}^{(p)} g_{r},
$$


we get $\varphi^{(p)}=g \psi^{(p)}$. Consequently,

$$
\frac{\varphi^{(p)} f_{i}}{f_{1}^{2}+\cdots+f_{r}^{2}}=\frac{\psi^{(p)} g_{i}}{g_{1}^{2}+\cdots+g_{r}^{2}} \quad \text { on } \Omega \backslash Z\left(f_{1}, \ldots, f_{r}\right) \text {. }
$$

Since the set $Z\left(f_{1}, \ldots f_{r}\right)$ is nowhere dense in $\Omega$, it follows that $\psi$ satisfies the PT for the $g_{i}$.

Lemma 2.2. Let $X$ be an irreducible nonsingular real algebraic variety and let $f_{1}, \ldots, f_{r}$ be continuous rational functions on $X$, not all identically equal to 0 . Then the Zariski closure of $Z\left(f_{1}, \ldots, f_{r}\right)$ is Zariski nowhere dense in $X$. In particular, $Z\left(f_{1}, \ldots, f_{r}\right)$ is Euclidean nowhere dense in $X$.

Proof. Setting $f=f_{1}^{2}+\cdots+f_{r}^{2}$, we get $Z(f)=Z\left(f_{1}, \ldots, f_{r}\right)$. The function $f$ is continuous rational and satisfies

$$
Z(f) \subset Z\left(\left.f\right|_{X \backslash P(f)}\right) \cup P(f),
$$

which implies both assertions.

Lemma 2.3. Let $X$ be an irreducible nonsingular real algebraic surface and let $f_{1}, \ldots, f_{r}$ be continuous rational functions on $X$. Then, for every point $p \in X$, there exists a Zariski open neighborhood $X^{(p)} \subset X$ of $p$ and there exist regular functions $g_{1}, \ldots, g_{r}, g, h$ on $X^{(p)}$ such that $Z(h) \neq X^{(p)}, Z\left(g_{1}, \ldots, g_{r}\right) \subset\{p\}$, and $h f_{i}=g g_{i}$ on $X^{(p)}$ for $i=1, \ldots, r$.

Proof. We can find regular functions $\lambda_{1}, \ldots, \lambda_{r}, \mu$ on $X$ such that $Z(\mu) \neq X$ and $f_{i}=\lambda_{i} / \mu$ on $X \backslash Z(\mu)$ for $i=1, \ldots, r$. Since $X$ is nonsingular, the local ring of regular functions at each point $p \in X$ is a unique factorization domain. Consequently, there exists a Zariski open neighborhood $X^{(p)} \subset X$ of $p$ and there exist regular functions $g_{1}, \ldots, g_{r}, g$ on $X^{(p)}$ such that $\lambda_{i}=g g_{i}$ on $X^{(p)}$ and $Z\left(g_{1}, \ldots, g_{r}\right) \subset\{p\}$. To complete the proof it suffices to set $h:=\left.\mu\right|_{X^{(p)}}$.

Lemma 2.4. Let $X$ be an irreducible nonsingular real algebraic surface and let $f_{1}, \ldots, f_{r}$ be continuous rational functions on $X$, not all identically equal to 0 . Let $\varphi$ be a continuous function on $X$ that satisfies the PT for the $f_{i}$. Then, for every point $p \in X$, there exists a Zariski open neighborhood $X^{(p)} \subset X$ of $p$ and there exist continuous functions $\alpha_{1}^{(p)}, \ldots, \alpha_{r}^{(p)}$ on $X^{(p)}$ and real numbers $c_{1}^{(p)}, \ldots, c_{r}^{(p)}$ such that

$$
\begin{gathered}
\varphi=\alpha_{1}^{(p)} f_{1}+\cdots+\alpha_{r}^{(p)} f_{r} \quad \text { on } X^{(p)}, \quad \text { and } \\
\alpha_{i}^{(p)}=c_{i}^{(p)}+\frac{\left(\varphi-\left(c_{1}^{(p)} f_{1}+\cdots+c_{r}^{(p)} f_{r}\right)\right) f_{i}}{f_{1}^{2}+\ldots+f_{r}^{2}} \quad \text { on } X^{(p)} \backslash Z\left(f_{1}, \ldots, f_{r}\right)
\end{gathered}
$$

for $i=1, \ldots, r$.

Proof. By Lemma 2.3, we can find a Zariski open neighborhood $X^{(p)} \subset X$ of $p$ and regular functions $g_{1}, \ldots, g_{r}, g, h$ on $X^{(p)}$ such that

$$
\begin{gathered}
Z\left(g_{1}, \ldots, g_{r}\right) \subset\{p\} \\
h f_{i}=g g_{i} \quad \text { on } X^{(p)} \quad \text { for } i=1, \ldots, r
\end{gathered}
$$

and $Z(h) \neq X^{(p)}$. Since $\varphi$ satisfies the PT for the $f_{i}$, it follows that $\left.h \varphi\right|_{X^{(p)}}$ satisfies the PT for the $\left.h f_{i}\right|_{X^{(p)}}=g g_{i}$. According to Lemma 2.2, the set

$$
Z\left(\left.h f_{1}\right|_{X^{(p)}}, \ldots,\left.h f_{r}\right|_{X^{(p)}}\right)=Z\left(g g_{1}, \ldots, g g_{r}\right)
$$


is nowhere dense in $X^{(p)}$. Hence, in view of Lemma 2.1, there exists a unique continuous function $\psi$ on $X^{(p)}$ such that

$$
\left.h \varphi\right|_{X^{(p)}}=g \psi
$$

Furthermore, $\psi$ satisfies the PT for the $g_{i}$. Consequently, taking (1) into account, we can write $\psi$ in the form

$$
\psi=\psi^{(p)}+c_{1}^{(p)} g_{1}+\cdots+c_{r}^{(p)} g_{r}
$$

where $c_{i}^{(p)} \in \mathbb{R}$ and the functions $B_{i}^{(p)}$ on $X^{(p)}$, defined by

$$
B_{i}^{(p)}=\frac{\psi^{(p)} g_{i}}{g_{1}^{2}+\cdots+g_{r}^{2}} \quad \text { on } X^{(p)} \backslash\{p\} \quad \text { and } \quad B_{i}^{(p)}(p)=0
$$

are continuous at $p$. It follows that the $B_{i}^{(p)}$ are continuous on $X^{(p)}$.

Defining $\varphi^{(p)}$ by

$$
\varphi=\varphi^{(p)}+c_{1}^{(p)} f_{1}+\cdots+c_{r}^{(p)} f_{r}
$$

and making use of (2)-(6), we get

$$
B_{i}^{(p)}=\frac{\varphi^{(p)} f_{i}}{f_{1}^{2}+\cdots+f_{r}^{2}} \quad \text { on } X^{(p)} \backslash(\{p\} \cup Z(g)) .
$$

By continuity,

$$
B_{i}^{(p)}=\frac{\varphi^{(p)} f_{i}}{f_{1}^{2}+\cdots+f_{r}^{2}} \quad \text { on } X^{(p)} \backslash Z\left(f_{1}, \ldots, f_{r}\right) .
$$

The functions $\alpha_{i}^{(p)}:=c_{i}^{(p)}+B_{i}^{(p)}$ are continuous on $X^{(p)}$ and in view of (6), (7) they satisfy

$$
\varphi=\alpha_{1}^{(p)} f_{1}+\cdots+\alpha_{r}^{(p)} f_{r} \quad \text { on } X^{(p)} \backslash Z\left(f_{1}, \ldots, f_{r}\right) .
$$

By continuity, the last equality holds on $X^{(p)}$. The proof is complete.

Proof of Theorem 1.5. By Lemma 2.4, a partition of unity argument completes the proof.

Lemma 2.4 contains more information than we needed for the proof of Theorem 1.5 . However, the full statement will be used to prove Theorem 1.6 in Section 3 .

\section{Continuous rational solutions}

We will frequently use, not necessarily explicitly referring to it, the following fact: If $X$ is a nonsingular real algebraic variety, $X^{0} \subset X$ a Zariski open subset, and $U \subset X$ a Euclidean open subset, then $X^{0} \cap U$ is Euclidean dense in $U$.

Lemma 3.1. Let $X$ be a nonsingular real algebraic variety, $\psi: X \rightarrow \mathbb{R}$ a regular function, and $f: X \backslash Z(\psi) \rightarrow \mathbb{R}$ a continuous rational function. Then there exists an integer $N_{0}>0$ such that for every integer $N \geq N_{0}$, the function $\psi^{N} f$, extended by 0 on $Z(\psi)$, is continuous rational on $X$. 
Proof. According to a variant of the Łojasiewicz inequality [2, Prop. 2.6.4], it suffices to prove that $f$ is a semialgebraic function. This is straightforward since the graph of the function $f$ restricted to $(X \backslash Z(\psi)) \backslash P(f)$ is a semialgebraic subset of $(X \backslash Z(\psi)) \times \mathbb{R}$, whose closure is equal to the graph of $f$.

Lemma 3.2. Let $X$ be a nonsingular real algebraic variety and let $\left\{X^{1}, \ldots, X^{m}\right\}$ be a Zariski open cover of $X$. Let $f_{1}, \ldots, f_{r}, \varphi$ be continuous rational functions on $X$ such that for $j=1, \ldots, m$ the restriction $\left.\varphi\right|_{X^{j}}$ can be written in the form

$$
\left.\varphi\right|_{X^{j}}=\left.\sum_{i=1}^{r} \varphi_{i j} f_{i}\right|_{X^{j}}
$$

where the $\varphi_{i j}$ are continuous rational functions on $X^{j}$. Then $\varphi$ can be written in the form

$$
\varphi=\sum_{i=1}^{r} \varphi_{i} f_{i},
$$

where the $\varphi_{i}$ are continuous rational functions on $X$ with

$$
P\left(\varphi_{i}\right) \subset \bigcup_{j=1}^{m}\left(P\left(\varphi_{i j}\right) \cup\left(X \backslash X^{j}\right)\right) .
$$

Proof. We may assume that $X$ is irreducible and the $X^{j}$ are all nonempty. Then each $X^{j}$ is Euclidean dense in $X$. We choose a regular function $\psi_{j}$ on $X$ with $Z\left(\psi_{j}\right)=X \backslash X^{j}$. By Lemma 3.1, there exists a positive integer $N$ such that the $\varphi_{i j}$ can be written as

$$
\varphi_{i j}=\frac{a_{i j}}{\psi_{j}^{N}} \quad \text { on } X^{j}
$$

where the $a_{i j}$ are continuous rational functions on $X$. It follows that

$$
\psi_{j}^{N} \varphi=\sum_{i=1}^{r} a_{i j} f_{i}
$$

on $X^{j}$. By continuity, (2) holds on $X$. Multiplying both sides of (2) by $\psi_{j}^{N}$ and summing over $j$, we get

$$
b \varphi=\sum_{i=1}^{r} b_{i} f_{i}
$$

where

$$
b=\sum_{j=1}^{m} \psi_{j}^{2 N} \quad \text { and } \quad b_{i}=\sum_{j=1}^{m} a_{i j} \psi_{j}^{N} .
$$

The function $b$ is regular with $Z(b)=\varnothing$, which implies that $\varphi_{i}:=b_{i} / b$ is a continuous rational function on $X$. In view of $(3)$ we have

$$
\varphi=\sum_{i=1}^{r} \varphi_{i} f_{i}
$$

By construction,

$$
P\left(\varphi_{i}\right) \subset \bigcup_{j=1}^{m} P\left(a_{i j}\right)
$$


while (1) implies

$$
P\left(a_{i j}\right) \subset P\left(\varphi_{i j}\right) \cup\left(X \backslash X^{j}\right)
$$

The proof is complete.

We will make use of rational maps and rational functions understood in the standard way. A rational map $F: X \rightarrow Y$, between real algebraic varieties $X$ and $Y$, is the equivalence class of regular maps with values in $Y$, defined on Zariski open dense subsets of $X$; two such regular maps $f_{1}: X^{1} \rightarrow Y$ and $f_{2}: X^{2} \rightarrow Y$ are declared to be equivalent if $\left.f_{1}\right|_{X^{0}}=\left.f_{2}\right|_{X^{0}}$ for some Zariski open dense subset $X^{0} \subset X^{1} \cap X^{2}$. We denote by $\operatorname{dom}(F)$ the union of all the domains of regular maps representing $F$. Thus $F$ determines a regular map $F: \operatorname{dom}(F) \rightarrow Y$. The polar set pole $(F):=X \backslash \operatorname{dom}(F)$ is Zariski nowhere dense in $X$. If $Y=\mathbb{R}$, then $F$ is called a rational function on $X$. The rational functions on $X$ form a ring (a field, if $X$ is irreducible), denoted $\mathbb{R}(X)$.

Definition 3.3. A rational function $R$ on a real algebraic variety $X$ is said to be locally bounded if for every point $p \in X$, one can find a Zariski open dense subset $X_{p} \subset X$, a Euclidean open neighborhood $U_{p} \subset X$ of $p$, and a real number $M_{p}>0$ such that

$$
|R(x)| \leq M_{p} \quad \text { for all } x \in U_{p} \cap \operatorname{dom}(R) \cap X_{p} .
$$

It readily follows that the set of all locally bounded rational functions on $X$ forms a subring of $\mathbb{R}(X)$.

Actually, Definition 3.3 would not be affected if we substituted for each $X_{p}$ the set $X^{\mathrm{ns}}$ of all nonsingular points of $\mathrm{X}$. Definition 3.3 imposes no restriction if the point $p$ is not in the Euclidean closure of $X^{\text {ns }}$.

Example 3.4. Consider the Whitney umbrella $W:=\left(x^{2}=y^{2} z\right) \subset \mathbb{R}^{3}$. The set of singular points of $W$ is the $z$-axis. The rational function $1 /(z+1)$ is locally bounded on $W$, but it is not locally bounded on the $z$-axis.

We will consider locally bounded rational functions only on nonsingular varieties. A typical example is the following.

Example 3.5. The rational function $x y /\left(x^{2}+y^{2}\right)$ on $\mathbb{R}^{2}$ is locally bounded (even bounded), but it cannot be extended to a continuous function on $\mathbb{R}^{2}$.

Lemma 3.6. Let $X$ be a nonsingular real algebraic variety and let $R$ be a locally bounded rational function on $X$. Then the polar set $\operatorname{pole}(R)$ is of codimension at least 2 .

Proof. Using the inclusion $\mathbb{R} \subset \mathbb{P}^{1}(\mathbb{R})$, we obtain a rational map $R^{*}: X \rightarrow \mathbb{P}^{1}(\mathbb{R})$ determined by $R$. The polar set pole $\left(R^{*}\right)$ is of codimension at least 2 [8, p. 129, Thm. 2.17]. Since $R$ is locally bounded, we have pole $(R)=\operatorname{pole}\left(R^{*}\right)$, which completes the proof.

Remark 3.7. Let $X$ be a nonsingular real algebraic variety. Any continuous rational function $f$ on $X$ determines a rational function $\tilde{f}$ on $X$, which is represented by the regular function $\left.f\right|_{X \backslash P(f)}$. Clearly, $P(f)=\operatorname{pole}(\tilde{f})$. Furthermore, if $g$ is a continuous rational function on $X$, not identically equal to 0 on any irreducible component of $X$, then the quotient $\tilde{f} / \tilde{g}$ is a well defined rational function on $X$ (see Lemma 2.2). To simplify notation, we will prefer to say "the rational function $f$ " or "the rational function $f / g$ " instead of writing $\tilde{f}$ or $\tilde{f} / \tilde{g}$, respectively. For the rational function $f / g$, we have

$$
\operatorname{pole}(f / g) \subset P(f) \cup P(g) \cup Z(g) .
$$


Lemma 3.8. Let $X$ be an irreducible nonsingular real algebraic variety and let $\varphi, f_{1}, \ldots, f_{r}$ be continuous rational functions on $X$, where the $f_{i}$ are not all identically equal to 0 . For $i=1, \ldots, r$ and $\mathbf{c}=\left(c_{1}, \ldots, c_{r}\right) \in \mathbb{R}^{r}$, let

$$
R_{\mathbf{c} i}:=\frac{\left(\varphi-\left(c_{1} f_{1}+\cdots+c_{r} f_{r}\right)\right) f_{i}}{f_{1}^{2}+\cdots+f_{r}^{2}} .
$$

If $\varphi$ satisfies the PT for $f_{1}, \ldots, f_{r}$, then each rational function $R_{\mathbf{c} i}$ is locally bounded on $X$.

Proof. Let $Z:=Z\left(f_{1}, \ldots, f_{r}\right)$ and let $S \subset X$ be an arbitrary subset. The $R_{\mathbf{c} i}$ are well defined functions on $X \backslash Z$. Setting $R_{i}=R_{\mathbf{0} i}$, where $\mathbf{0}=(0, \ldots, 0) \in \mathbb{R}^{r}$, we get

$$
R_{\mathbf{c} i}=R_{i}-\frac{c_{1} f_{i} f_{1}+\cdots+c_{r} f_{i} f_{r}}{f_{1}^{2}+\cdots+f_{r}^{2}}
$$

Since

$$
\left|\frac{f_{i} f_{j}}{f_{1}^{2}+\cdots+f_{r}^{2}}\right| \leq \frac{1}{2} \quad \text { on } X \backslash Z
$$

it follows that $R_{\mathbf{c} i}$ is bounded on $S \cap(X \backslash Z)$ if and only if $R_{i}$ is such.

Suppose that $\varphi$ satisfies the PT for $f_{1}, \ldots, f_{r}$, fix a point $p \in X$, and set $Z^{(p)}=Z \cup\{p\}$. The function $\varphi$ can be written in the form

$$
\varphi=\varphi^{(p)}+c_{1}^{(p)} f_{1}+\cdots+c_{r}^{(p)} f_{r},
$$

where $c_{i}^{(p)} \in \mathbb{R}$ and the functions $A_{i}^{(p)}$ on $X$, defined by

$$
A_{i}^{(p)}=\frac{\varphi^{(p)} f_{i}}{f_{1}^{2}+\cdots+f_{r}^{2}} \quad \text { on } X \backslash Z^{(p)} \quad \text { and } \quad A_{i}^{(p)}=0 \quad \text { on } Z^{(p)},
$$

are continuous at $p$. In particular, the $A_{i}^{(p)}$ are bounded on some Euclidean open neighborhood $U_{p} \subset X$ of $p$. Consequently, the functions $R_{\mathbf{c}^{(p)} i}$, where $\mathbf{c}^{(p)}=\left(c_{1}^{(p)}, \ldots, c_{r}^{(p)}\right)$, are bounded on $U_{p} \cap(X \backslash Z)$, which in turn implies that the $R_{i}$ are bounded on $U_{p} \cap(X \backslash Z)$. This conclusion remains valid if $Z$ is replaced by its Zariski closure $V$ in $X$. The proof is complete since the subset $X \backslash V \subset X$ is Zariski open dense by Lemma 2.2.

Proof of Theorem 1.6. It suffices to prove that (b) implies (a) together with the extra conditions stipulated on the $\varphi_{i}$. Let us suppose that (b) holds. We may assume that $X$ is irreducible and the $f_{i}$ are not all identically equal to 0 . According to Lemma 2.4, for each point $p \in X$, we can find a Zariski open neighborhood $X^{(p)} \subset X$ of $p$ and continuous functions $\alpha_{1}^{(p)}, \ldots, \alpha_{r}^{(p)}$ on $X^{(p)}$ such that

$$
\varphi=\alpha_{1}^{(p)} f_{1}+\cdots+\alpha_{r}^{(p)} \quad \text { on } X^{(p)}
$$

and

$$
\alpha_{i}^{(p)}=c_{i}^{(p)}+R_{i}^{(p)} \quad \text { on } X^{(p)} \backslash Z,
$$

where $Z=Z\left(f_{1}, \ldots, f_{r}\right), c_{i}^{(p)} \in \mathbb{R}$, and

$$
R_{i}^{(p)}=\frac{\left(\varphi-\left(c_{1}^{(p)} f_{1}+\cdots+c_{r}^{(p)} f_{r}\right)\right) f_{i}}{f_{1}^{2}+\cdots+f_{r}^{2}} \quad \text { on } X^{(p)} \backslash Z .
$$

We regard the $R_{i}^{(p)}$ as rational functions on $X$ and set

$$
X_{0}^{(p)}:=\operatorname{dom}\left(R_{1}^{(p)}\right) \cap \ldots \cap \operatorname{dom}\left(R_{r}^{(p)}\right) .
$$


Clearly,

$$
X \backslash X_{0}^{(p)} \subset Z
$$

Furthermore, according to Lemmas 3.6 and 3.8, $X \backslash X_{0}^{(p)}$ is a finite set. Since the set $X^{(p)} \backslash Z$ is Euclidean dense in $X$ (see Lemma 2.2), it follows that

$$
\alpha_{i}^{(p)}=R_{i}^{(p)} \quad \text { on } X^{(p)} \cap X_{0}^{(p)} .
$$

Consequently, we obtain a well defined continuous rational function $\beta_{i}^{(p)}$ on $X_{1}^{(p)}:=X^{(p)} \cup X_{0}^{(p)}$ by setting

$$
\beta_{i}^{(p)}=\alpha_{i}^{(p)} \quad \text { on } X^{(p)} \quad \text { and } \quad \beta_{i}^{(p)}=c_{i}^{(p)}+R_{i}^{(p)} \quad \text { on } X_{0}^{(p)} .
$$

By construction,

$$
\varphi=\beta_{1}^{(p)} f_{1}+\cdots+\beta_{r}^{(p)} f_{r} \quad \text { on } X_{1}^{(p)} .
$$

Now it is easy to complete the proof. We choose a finite collection of points $p_{1}, \ldots, p_{m}$ in $X$ so that the sets $X^{j}:=X_{1}^{\left(p_{j}\right)}$ form a cover of $X$. Setting $\varphi_{i j}:=\beta_{i}^{\left(p_{j}\right)}$, we get

$$
\left.\varphi\right|_{X^{j}}=\left.\sum_{i=1}^{r} \varphi_{i j}\right|_{X^{j}}, \quad P\left(\varphi_{i j}\right) \subset\left(P(\varphi) \cup Z \cup P\left(f_{1}\right) \cup \ldots \cup P\left(f_{r}\right)\right) \cap\left(X \backslash X_{0}^{\left(p_{j}\right)}\right) .
$$

By Lemma 3.2 , there exist continuous rational functions $\varphi_{1}, \ldots, \varphi_{r}$ on $X$ such that

$$
\varphi=\varphi_{1} f_{1}+\cdots+\varphi_{r} f_{r}
$$

and

$$
P\left(\varphi_{i}\right) \subset \bigcup_{j=1}^{r}\left(P\left(\varphi_{i j}\right) \cup\left(X \backslash X^{j}\right)\right)
$$

The functions $\varphi_{i}$ satisfy all the requirements.

\section{References}

[1] M. Bilski, W. Kucharz, A. Valette, and G. Valette, Vector bundles and reguluous maps, Math. Z. 275 (2013), 403-418.

[2] J. Bochnak, M. Coste, and M.-F. Roy, Real Algebraic Geometry, Ergeb. der Math. und ihrer Grenzgeb. Folge 3, vol. 36, Springer, 1998.

[3] H. Brenner, Continuous solutions to algebraic forcing equations, arXiv:0608611 [math.AC].

[4] N. Epstein and M. Hochster, Continuous closure, axes closure, and natural closure, arXiv:1106.3462v2 [math.AC].

[5] C. Fefferman and J. Kollár, Continuous solutions of linear equations, From Fourier analysis and number theory to Radon transforms and geometry, 233-282, Dev. Math. 28, Springer, 2013.

[6] G. Fichou, J. Huismann, F. Mangolte, and J.-Ph. Monnier, Fonctions régulues, arXiv:1112.3800 [math.AG], to appear in J. Reine Angew. Math. 
[7] G. Fichou, J.-Ph. Monnier, and R. Quarez, Continuous functions in the plane regular after one blowing-up, arXiv:1409.8223 [math.AG].

[8] S. Iitaka, Algebraic Geometry. An Introduction to Birational Geometry of Algebraic Varieties. Springer, 1982.

[9] J. Kollár, Continuous closure of sheaves, Michigan Math. J. 61 (2012), 475-491.

[10] J. Kollár and K. Nowak, Continuous rational functions on real and $p$-adic varieties, Math. Z. 279 (2015), 85-97.

[11] W. Kucharz, Rational maps in real algebraic geometry, Adv. Geom. 9 (2009), 517-539.

[12] W. Kucharz, Regular versus continuous rational maps, Topology Appl. 160 (2013), 13751378 .

[13] W. Kucharz, Approximation by continuous rational maps into spheres, J. Eur.Math. Soc. 16 (2014), 1555-1569.

[14] W. Kucharz, Continuous rational maps into the unit 2-sphere, Arch. Math. (Basel) 102 (2014), 257-261.

[15] W. Kucharz, Continuous rational maps into spheres, arXiv:1403.5127 [math.AG].

[16] W. Kucharz and K. Kurdyka, Stratified-algebraic vector bundles, arXiv:1308.4376 [math.AG], to appear in J. Reine Angew. Math.

[17] W. Kucharz and K. Kurdyka, Curve-rational functions, arXiv:1509.05905 [math.AG].

[18] W. Kucharz and K. Kurdyka, Comparison of stratified-algebraic and topological Ktheory, arXiv:1511.04238 [math.AG].

[19] K.J. Nowak, Algebraic geometry over Henselian rank one valued fields, arXiv:1410.3280 [math.AG].

[20] M. Zieliński, Homotopy properties of some real algebraic maps, to appear in Homology, Homotopy and Applications.

Wojciech Kucharz

Institute of Mathematics

Faculty of Mathematics and Computer Science

Jagiellonian University

Łojasiewicza 6

30-348 Kraków

Poland

E-mail address: Wojciech.Kucharz@im.uj.edu.pl

Krzysztof Kurdyka

Laboratoire de Mathématiques

UMR $5175 \mathrm{du}$ CNRS

Université Savoie Mont Blanc

Campus Scientifique

73376 Le Bourget-du-Lac Cedex

France

E-mail address: kurdyka@univ-savoie.fr 